\title{
Applicable Formulas for Shear and Thermal Buckling of Perforated Rectangular Panels
}

\author{
Husam Al Qablan \\ Civil Engineering Department, Faculty of Engineering, The Hashemite University, Zarqa 13133, P.O. Box 330127, Jordan \\ Correspondence should be addressed to Husam Al Qablan; hqablan@gmail.com
}

Received 11 November 2021; Accepted 6 January 2022; Published 19 January 2022

Academic Editor: Ying Qin

Copyright (C) 2022 Husam Al Qablan. This is an open access article distributed under the Creative Commons Attribution License, which permits unrestricted use, distribution, and reproduction in any medium, provided the original work is properly cited.

The goal of this study is to create semiempirical formulations for predicting thermal and shear buckling loads of perforated rectangular isotropic panels for eight combinations of boundary conditions. The finite element method (FEM) was used to develop and evaluate empirical formulations. In this study, the influences of plate aspect ratios, boundary conditions, and perforation sizes on the buckling strength of perforated panels subjected to shear and thermal loads are investigated. The proposed formulas will enable consistent and reliable computation of the buckling loads for perforated rectangular panels without the need for complex calculations. The results of the empirical equations were found to be reasonably consistent with the outcomes of finite element analysis (FE) and findings from the literature. Various perforation patterns are investigated, ranging from a single circular hole up to 441 circular holes distributed across the plate. The results show that plates with a single central cutout have lower shear buckling loads than plates with multiple holes and an equal total cutout area.

\section{Introduction}

Rectangular plate buckling is an important aspect of structural design, especially if lightweight design is the primary goal. Plates with perforations are frequently used to construct a lightweight structure or opening for accessibility reasons such as heating, plumbing, electrical systems, and maintenance. The presence of these cutouts alters the stiffness and the stress distribution of the panel, potentially changing the buckling failure mode [1-5]. Earlier investigations on the buckling of perforated panels focused on the effects of loading instances, boundary conditions, hole size, shape, number, and position on the buckling pattern of rectangular plates.

The foundation of reliable structural designs is a thorough understanding of buckling stresses and associated mode shapes, and establishing an easy-to-implement method can provide an appropriate approach to it. Due to the intricacy of the problem, most researchers studied the buckling conduct of perforated isotropic simply supported rectangular panels utilizing the FEM and/or the Rayleigh-Ritz method [1, 4, 6-12]. For example, the FEM was employed by [1] to study the buckling performance of isotropic simply supported rectangular plates with rectangular/circular perforations postured at various locations on the panel exhibited to in-plane stresses [9]. Investigate the buckling behavior for rectangular steel panels with eccentric circular perforations exposed to uniaxial compression loads. The FEM was also used to explore the buckling behavior of perforated composite plates subjected to in-plane loads $[2,11,13,14]$. They investigated the impact of perforation types, locations, and sizes on the buckling behavior of the composite plates.

The analysis of shear buckling and thermal buckling of thin plates has piqued the interest of numerous researchers in recent years $[2,3,15-20]$. For isotropic square panels and thin-walled channel sections with circular and square perforations, [3] used the FEM and the Spline Finite Strip Method to calculate the shear buckling force. For design reasons, he also gives approximation equations for shear buckling coefficients. Amabili and Tajahmadi [15] investigate experimentally the postbuckling behavior of isotropic and laminated clamped edge plates exposed to thermal variations, while [18] used the finite element method to 
study the thermal buckling behavior of isotropic and laminated composite plates with different boundary conditions [19]. Investigate the mechanical and thermal buckling analysis of functionally graded panels using the element-free kp-Ritz method.

This study investigates the buckling demeanor of perforated isotropic rectangular plates with various boundary conditions exhibited to shear and thermal load conditions. The primary goal of this study is to develop a set of efficient closed-form semiempirical equations that can be used to quickly analyse and predict buckling loads for isotropic rectangular perforated panels without requiring extensive calculations. The suggested formulas would make calculating buckling loads quick and easy while maintaining reasonable accuracy. In addition, this research will produce a set of data that could be used in practical examinations of the buckling behavior of rectangular plates for eight combinations of boundary conditions having circular/central perforations. To validate the veracity of the suggested semiempirical formulations, the results of the suggested equations, the FEM, and the available literature findings were contrasted.

\section{Problem Formulation}

For isotropic rectangular panels exhibited to shear force, the critical buckling load can be expressed as [21]

$$
N_{\text {critical }}=-K_{\text {Shear }} \frac{E h^{3} \pi^{2}}{12 b^{2}\left(v^{2}-1\right)},
$$

where $K_{\text {Shear }}, E, v, b$, and $h$ represent the shear buckling coefficient, modulus of elasticity, Poisson's ratios, panel width, and panel thickness, respectively. Finding closedform solutions for the buckling of rectangular isotropic panels with central/circular perforation subjected to shear loads or thermal loads is a challenging task. Based on the investigation of the buckling behavior of various perforated rectangular isotropic panels utilizing the FEM and accessible literature data, semiempirical formulations were constructed to solve this dilemma, as demonstrated in the following equations:

$$
\begin{aligned}
& N_{\text {critical }}=-K_{\text {Shear }} \frac{E h^{3} \pi^{2}}{12(b+B)^{2}\left(v^{2}-1\right)} . \\
& T_{\text {critical }}=T_{\text {cr }} R,
\end{aligned}
$$

where $B$ is the rectangular perforated plate's critical load adjustment factor for shear buckling, $T_{\text {critical }}$ is the critical temperature for perforated rectangular plate, $T_{\mathrm{cr}}$ is the critical temperature for rectangular plate buckling, and $R$ is the rectangular perforated plate's critical load adjustment factor for thermal buckling. Adding the adjustment factors to the plate width was initially used and presented by [5] for buckling of perforated simply and clumped supported square laminated plates subjected to uniaxial and biaxial loads. Herein, the optimized solutions for the buckling load adjustment factors ( $B$ and $R$ ), the shear buckling coefficients $K_{\text {Shear }}$, and and the minimum critical temperature $T_{\text {cr }}$ were found using the Generalized Reduced Gradient (GRG) method. The GRG is a nonlinear optimization approach built into the Excel Solver feature in Microsoft Office for nonlinear optimization. To assort the variable parameters into $K_{\text {Shear }}, T_{\mathrm{cr}}$, and the adjustment factors ( $B$ and $R$ ), the GRG algorithm separated the parameters set into two categories (basic and nonbasic parameters). The adjustment factors $(B)$ altered the buckling load of the isotropic rectangular panels by increasing/decreasing the plate area with a rise in the perforation diameter. The length/width panel proportion $(a / b)$, the boundary conditions, and the proportion of cutout diameter to panel width $(d / b)$ all had a role in the increase/decrease in plate area. The adjustment factor $(R)$ altered the thermal buckling loads by increasing the thermal buckling loads with the rise in the perforation diameter.

Eight combinations of simply supported $(S)$, clamped $(C)$, and free $(F)$ edges are investigated to evaluate the effect of boundary conditions on the shear buckling loads. These combinations include SSSS, CCCC, CCSS, SSCC, FFCC, CCFF, SSFF, and FFSS. The abbreviation CCSS for example refers to the clamped supported boundary conditions of the two edges parallel to the $y$-axis and the simply supported boundary conditions for the two edges parallel to the $x$-axis as illustrated in Figure 1.

To find the optimal solutions for the shear and thermal buckling of perforated rectangular isotropic plates, initially, for each boundary condition, the exact numerical values based on the FE results for the buckling load adjustment factors ( $B$ and $R$ ), the shear buckling coefficients $K_{\text {Shear }}$, and the minimum critical temperature $T_{\mathrm{cr}}$ were determined using the Generalized Reduced Gradient (GRG) method. After determining the exact values for $K_{\text {Shear }}, T_{\text {cr }}, B$, and $R$, a simplified mathematical expression similar in general shape to published expression (if available) was generated to represent and link the values of $B, R, T_{\mathrm{cr}}$, and $K_{\text {Shear }}$ for different aspect ratios $(a / b)$ for each boundary condition. Linking these values for different aspect ratios $(a / b)$ is not an easy task. It requires many iterations and modifications of the mathematical formulas to minimize the errors. The accuracy of the empirical equations is then measured using the coefficient of determination $\left(R^{2}\right)$. Following the selection of mathematical expression (as a function of plate shape properties $(a, b, d$, and $K_{\text {Shear }}$ ) for each boundary condition, the fitness of each individual is evaluated. The mathematical expressions are then manually modified according to fitness with initial guesses for the constants in the equations. Those constants were modified using the GRG method to minimize the errors. The selection of mathematical expressions and constants is based on fitness to regenerate with an alteration. This process is repeated manually until the optimal solution is found in a simplified applicable form as shown in Tables 1 and 2. In this study, a large database including 126 and 112 data points for each boundary condition was used to develop the empirical formulas for shear and thermal buckling of the perforated plates, respectively. $85 \%$ of the data points were used to develop the empirical equations, while $15 \%$ of the data points were used to 

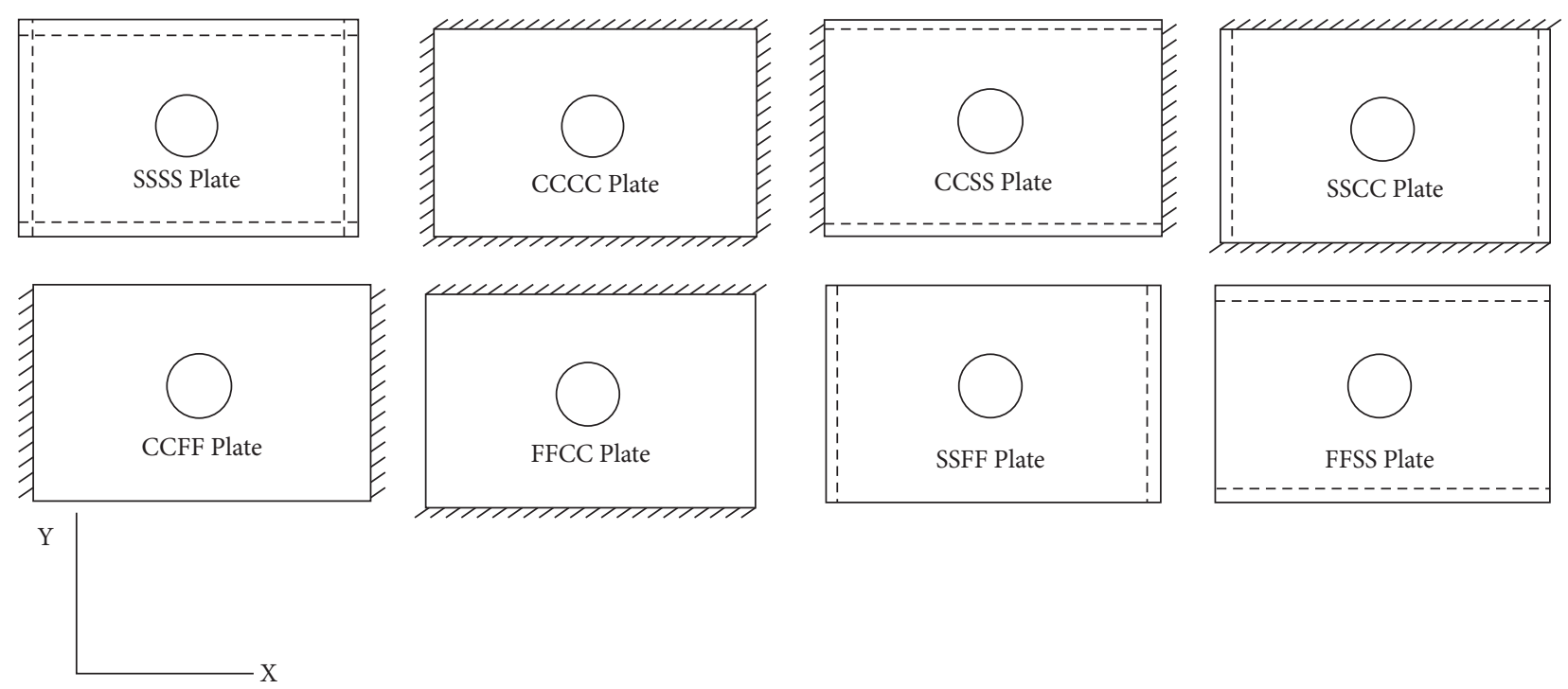

FIGURE 1: Boundary conditions of the plate.

TABLE 1: Shear buckling coefficients $K_{\text {Shear }}$ and buckling load modification factor $B$ for perforated isotropic rectangular plates.

\begin{tabular}{lcc}
\hline BC & $K_{\text {Shear }}$ & $B$ \\
\hline SSSS & $5.34+\frac{4}{(a / b)^{2}}[22]$ & $\left(\left(4.3 *\left(\frac{a}{b}\right)^{-0.3}\right) *\left(\frac{d}{b}\right)^{2}+\left(\frac{d}{b}\right) * \frac{K_{\text {Shear }}}{(12(a / b))} * \frac{b}{2}\right.$ \\
CCCC & $8.89+\frac{5.6}{(a / b)^{2}}[22]$ & $\left(2.4 *\left(\frac{d}{b}\right)^{2}+\left(\frac{d}{b}\right) * \frac{K_{\text {Shear }}}{(12(a / b))}\right) \frac{b}{2}$ \\
CCSS & $5.34+\frac{2.31}{(a / b)}-\frac{3.44}{(a / b)^{2}}+\frac{8.39}{(a / b)^{3}}[22]$ & $\left(3.2 *\left(\frac{d}{b}\right)^{2}+\left(\frac{d}{b}\right) * \frac{K_{\text {Shear }}}{(12(a / b))}\right) \frac{b}{2}$ \\
SSCC & $8.98+\frac{5.61}{(a / b)^{2}}-\frac{1.99}{(a / b)^{3}}[22]$ & $\left(\left(2+\frac{1}{(a / b)}\right) *\left(\frac{d}{b}\right)^{2}+\left(\frac{d}{b}\right) * \frac{K_{\text {Shear }}}{(12(a / b)}\right) * \frac{b}{2}$ \\
FFCC & $8.5-\left(\frac{a}{b}\right)^{-0.82}$ & $\left(1.3 *\left(1.9+\left(\frac{a}{b}\right)^{-2.3}\right)\left(\frac{d}{b}\right)^{2}-\frac{(d / b)}{4}\right) * \frac{b}{2}$ \\
CCFF & $7.85 *\left(\frac{a}{b}\right)^{-2.2}$ & $\left(\left(3.3 *\left(\frac{a}{b}\right)^{-0.68}\right)\left(\frac{d}{b}\right)^{2}-\frac{(d / b)}{4}\right) * \frac{b}{2}$ \\
SSFF & $4.0 *\left(\frac{a}{b}\right)^{-2.22}$ & $\left(\left(3.3 *\left(\frac{a}{b}\right)^{-0.68}\right)\left(\frac{d}{b}\right)^{2}-\frac{(d / b)}{4}\right) * \frac{b}{2}$ \\
FFSS & $5.22-\left(\frac{a}{b}\right)^{-0.3}$ & $\left(1.3 *\left(1.82+\left(\frac{a}{b}\right)^{-2.3}\right)\left(\frac{d}{b}\right)^{2}-\frac{(d / b)}{4}\right) * \frac{b}{2}$ \\
\hline
\end{tabular}

TAвLe 2: Minimum critical temperature $T_{\mathrm{cr}}$ and thermal buckling load adjustment factor $R$ for perforated isotropic rectangular plates.

\begin{tabular}{lcr}
\hline BC & $T_{\text {cr }}$ & $R$ \\
\hline SSSS & $\frac{\left(a^{2}+b^{2}\right) h^{2} \pi^{2}}{12 a^{2} b^{2} \alpha(1+v)}[23]$ & $1+\left(\frac{0.85}{(a / b)^{a / b}} * \frac{\pi d^{2}}{4}\right)^{2}$ \\
CCCC & $\frac{\left(3 a^{4}+2 a^{2} b^{2}+3 b^{4}\right) h^{2} \pi^{2}}{9 b^{2}\left(a^{4}+a^{2} b^{2}\right) \alpha(1+v)}[23]$ & $1+\left(\frac{1.5}{(a / b)^{1.7}} * \frac{\pi d^{2}}{4}\right)^{2}$ \\
CCSS & $\frac{\left(3 a^{4}+8 a^{2} b^{2}+16 b^{4}\right) h^{2} \pi^{2}}{12 b^{2}\left(3 a^{4}+4 a^{2} b^{2}\right) \alpha(1+v)}$ & $1+\left(\frac{(a / b)^{b / a}}{(a / b)^{2}} * \frac{\pi d^{2}}{4}\right)^{2}$ \\
SSCC & $\frac{\left(16 a^{4}+8 a^{2} b^{2}+3 b^{4}\right) h^{2} \pi^{2}}{12 b^{2}\left(4 a^{4}+3 a^{2} b^{2}\right) \alpha(1+v)}$ & $1+\left(\frac{(a / b)^{b / a}}{(a / b)^{2}} * \frac{\pi d^{2}}{4}\right)^{2}$ \\
CCFF & $\frac{h^{2} \pi^{2}}{3 a^{2} \alpha}\left(1+\frac{v^{3}}{3}\right)$ & $1+\left(\frac{(a / b)^{b / a}}{2 a / b} * \frac{\pi d^{2}}{4}\right)^{2}$ \\
SSFF & $\frac{h^{2} \pi^{2}}{12 a^{2} \alpha}\left(1-\frac{v^{3}}{3}\right)$ & $1+\left(\frac{(a / b)^{b / a}}{(3 a / b)^{2}} * \frac{\pi d^{2}}{4}\right)^{2}$ \\
\hline
\end{tabular}

validate the equations. These empirical equations are only valid for thin plates $(b / h>20)$. In addition, these empirical equations are limited for $a / b<10$. It has not been tested or validated for plates with a large aspect ratio between length to width $a / b>10$. Farther future instigations are required to validate and modify the empirical formulas for $a / b>10$. 


\section{Examples and Discussions}

3.1. Shear Buckling of Perforated Isotropic Plates. The critical loads of perforated panels exposed to shear loading were predicted using the proposed semiempirical equations as listed in the preceding section (see equation (2) and Table 1). The buckling analysis of the rectangular panels with circular/ central cutouts was carried out using the nonlinear FE program (ABAQUS). The FE model consisted of four noded quadrilateral shell elements with reduced integration. Figure 2 shows how the perforated panels were separated into a manageable number of elements. Preliminary runs were carried out to ensure that the findings were in convergence. The results of the semiempirical equation (see equation (2)) were compared to the literature's accessible results and the nonlinear FE's results. The proposed equations for calculating buckling loads are shown to be effective in the various applications detailed in the following.

In this study, the width dimensions $(b)$ of the panels were chosen to be $(b=2000 \mathrm{~mm})$. Nine different values of length to width ratios $(a / b)$ for the panels were used $(a / b=1.0, a /$ $b=1.5, a / b=2.0, a / b=2.5, a / b=3.0, a / b=3.5, a / b=4, a /$ $b=6$, and $a / b=10)$. The panels' thickness was chosen to be $h=12 \mathrm{~mm}$. Different values for the cutout dimeters $(d)$ were used in this study. Poisson's ratio $=0.3$ and Young's modulus $E=200 \mathrm{GPa}$ were chosen as isotropic material parameters (structural steel plate).

Tables 3 and 4 show the normalized buckling load $\left(\bar{N}_{\mathrm{cr}}=N_{\mathrm{cr}} b^{2} / \pi^{2} D\right)$ for square $(a / b=1.0)$ simply supported perforated plate case (SSSS) and clamped supported case (CCCC) subjected to shear loads respectively, where $D=$ $\mathrm{Eh}^{3} / 12\left(1-v^{2}\right)$ (flexural rigidity). These tables summarize the findings available from the literature, as well as the FE outcomes and the suggested empirical equation (see equation (2)). Figures 3 and 4 show the normalized critical loads for rectangular perforated isotropic plates with different length-width $(a / b)$ proportions. In these figures, the boundary conditions for the rectangular plates were chosen to be SSSS and CCCC, respectively. Table 5 shows the normalized critical loads $\left(\bar{N}_{\mathrm{cr}}=N_{\mathrm{cr}} b^{2} / \pi^{2} D\right)$ for perforated rectangular panels having CCSS, SSCC, CCFF, FFCC, SSFF, and FFSS boundary conditions.

The results in those figures and tables were obtained from the proposed equation (see equation (2)), available outcomes from the literature, and compared with results obtained from the finite element. The results demonstrate the capacity of the suggested formulas to estimate shear buckling loads for isotropic perforated rectangular panels with different boundary conditions. Furthermore, the perforation dimension, the boundary condition of the panels, and the aspect ratio length/width of the panel $(a / b)$ all have a substantial impact on the shear buckling stress, as seen in the figures and the tables.

Figure 5 depicts the increase's proportion in the width of the rectangular perforated isotropic panels with different boundary conditions subjected to shear loads. It is obvious that as the aspect ratio length/width of the panel $(a / b)$ rises, the width ratio $((b+B) / b)$ reduces for all boundary conditions with different percentages, which means that as the

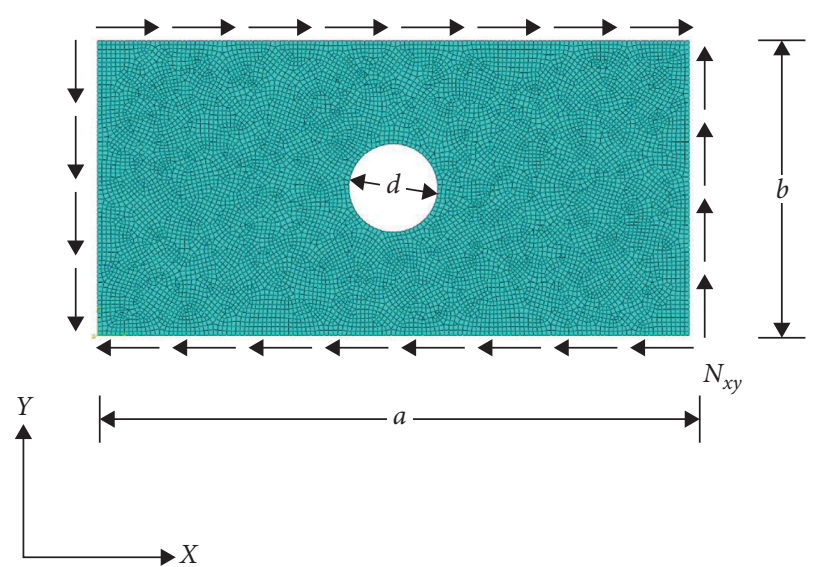

FIgURE 2: Undeformed shape for a rectangular plate with a central circular perforation.

plate length/width proportion $(a / b)$ increases, the perforation's influence on the buckling load decreases. For example, the reduction in shear buckling load for square perforated plate $(d / b=0.6, a / b=1.0)$ relative to a square plate without perforation is equal $75 \%$ for SSSS, $69 \%$ for CCCC, $72 \%$ for CCSS, $71 \%$ for SSCC, $60 \%$ for FFCC, $57 \%$ for CCFF, $57 \%$ for SSFF, and 57\% for FFSS boundary conditions. The reduction in shear buckling load for rectangular perforated plate $(d /$ $b=0.6, a / b=4.0)$ relative to a rectangular plate without cutout is equal to $57 \%$ for SSSS, $55 \%$ for CCCC, $62 \%$ for CCSS, $53 \%$ for SSCC, $52 \%$ for FFCC, $25 \%$ for CCFF, $25 \%$ for SSFF, and $48 \%$ for FFSS boundary conditions. The effect of the perforation size concerning the boundary conditions can also be seen in the figure. For example, the effect of the perforation sizes is more crucial for the SSSS panels than SSFF panels. It can also be noted from the figure that the perforation dimensions, particularly for large cutout dimensions, have a significant impact on reducing shear buckling. It is worth noting that the effect of small sizes of perforations $(d / b \leq 0.2)$ on the shear buckling loads can be ignored for plates having FFCC, CCFF, SSFF, and FFSS boundary conditions.

Figure 6 depicts the first mode shape for an isotropic rectangular panel with different boundary conditions exposed to shear loads $(a / b=1.5, d / b=0.2)$. Variations in displacement contour lines (mode shape) are strongly influenced by the boundary conditions, as shown in the figure, which explains why the buckling load reduces as the stiffness of the boundary conditions decreases. The CCCC boundary conditions, for example, achieved the highest buckling load values, while the SSFF boundary conditions achieved the lowest buckling load values, as can be seen in Figures 3 and 4 and Table 5 .

The effect of perforation patterns on shear buckling loads has been studied using the finite element method. Different percentages of cutout areas were chosen ranging from $5 \%$ up to $50 \%$ of the square simply supported and clamped supported isotropic plate area $(2 \mathrm{~m} \times 2 \mathrm{~m} \times 0.01 \mathrm{~m})$. Various perforation patterns are investigated, varying from a single circular hole up to 441 circular holes distributed across the plate of equal total cutout area for each cutout area 
TABLE 3: Normalized buckling loads for square $(a / b=1.0)$ simply supported (SSSS) perforated panel exposed to shear load case.

\begin{tabular}{|c|c|c|c|c|c|c|}
\hline$d / b$ & SFSM [3] & FEM [3] & Pham equations [3] & Reference [4] & FEM [4] & Present equation (2) \\
\hline 0 & 9.328 & 9.328 & 9.328 & 9.235 & 9.24 & 9.340 \\
\hline 0.05 & 9.059 & 9.125 & 8.997 & & & 8.893 \\
\hline 0.1 & 8.487 & 8.562 & 8.47 & 8.683 & 8.479 & 8.306 \\
\hline 0.15 & 7.737 & 7.797 & 7.747 & & & 7.625 \\
\hline 0.2 & 6.938 & 6.998 & 6.828 & 7.054 & 6.948 & 6.895 \\
\hline 0.25 & 6.153 & 6.199 & 6.122 & & & 6.157 \\
\hline 0.3 & 5.409 & 5.445 & 5.382 & 5.465 & 5.403 & 5.440 \\
\hline 0.35 & 4.718 & 4.753 & 4.704 & & & 4.768 \\
\hline 0.4 & 4.09 & 4.121 & 4.086 & 4.089 & 4.051 & 4.153 \\
\hline 0.45 & 3.53 & 3.556 & 3.528 & & & 3.601 \\
\hline 0.5 & 3.038 & 3.059 & 3.032 & 2.998 & 2.969 & 3.113 \\
\hline 0.55 & 2.612 & 2.629 & 2.596 & & & 2.687 \\
\hline 0.6 & 2.247 & 2.25 & 2.239 & 2.185 & 2.162 & 2.318 \\
\hline 0.65 & 1.936 & 1.94 & 1.959 & & & 1.999 \\
\hline 0.7 & 1.672 & 1.676 & 1.679 & & & 1.726 \\
\hline 0.75 & 1.449 & 1.446 & 1.399 & & & 1.493 \\
\hline 0.8 & 1.26 & 1.251 & 1.119 & & & 1.293 \\
\hline
\end{tabular}

TABLE 4: Normalized buckling loads for square $(a / b=1.0)$ clamped supported (CCCC) perforated panel exposed to shear load case.

\begin{tabular}{lcccc}
\hline$d / b$ & Reference $[4]$ & Reference [4] $(\mathrm{FE})$ & Present equation $(2)$ & Present $(\mathrm{FE})$ \\
\hline 0 & 14.371 & 14.358 & 14.464 & 14.662 \\
0.1 & 13.317 & 13.015 & 12.577 & 13.286 \\
0.2 & 10.785 & 10.693 & 10.589 & 10.898 \\
0.3 & 8.662 & 8.535 & 7.703 & 8.749 \\
0.4 & 6.853 & 6.701 & 5.637 & 6.931 \\
0.5 & 5.158 & 5.292 & 4.493 & 5.521 \\
0.6 & 4.059 & 4.264 & 4.505 \\
\hline
\end{tabular}
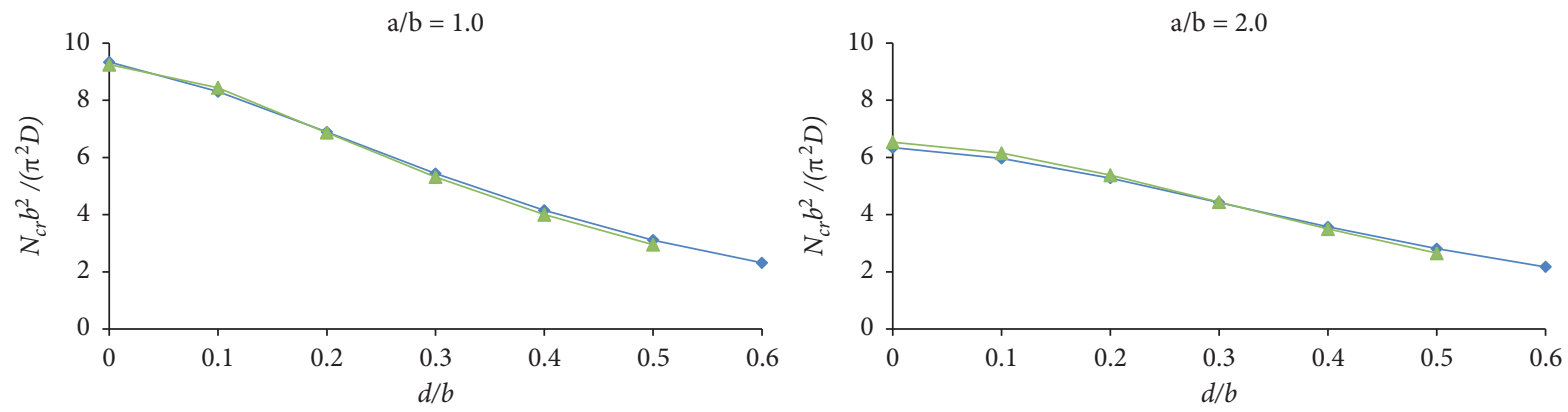

$\rightarrow$ Equation [2]

4- Yidris \& Hassan (2019) (FE)
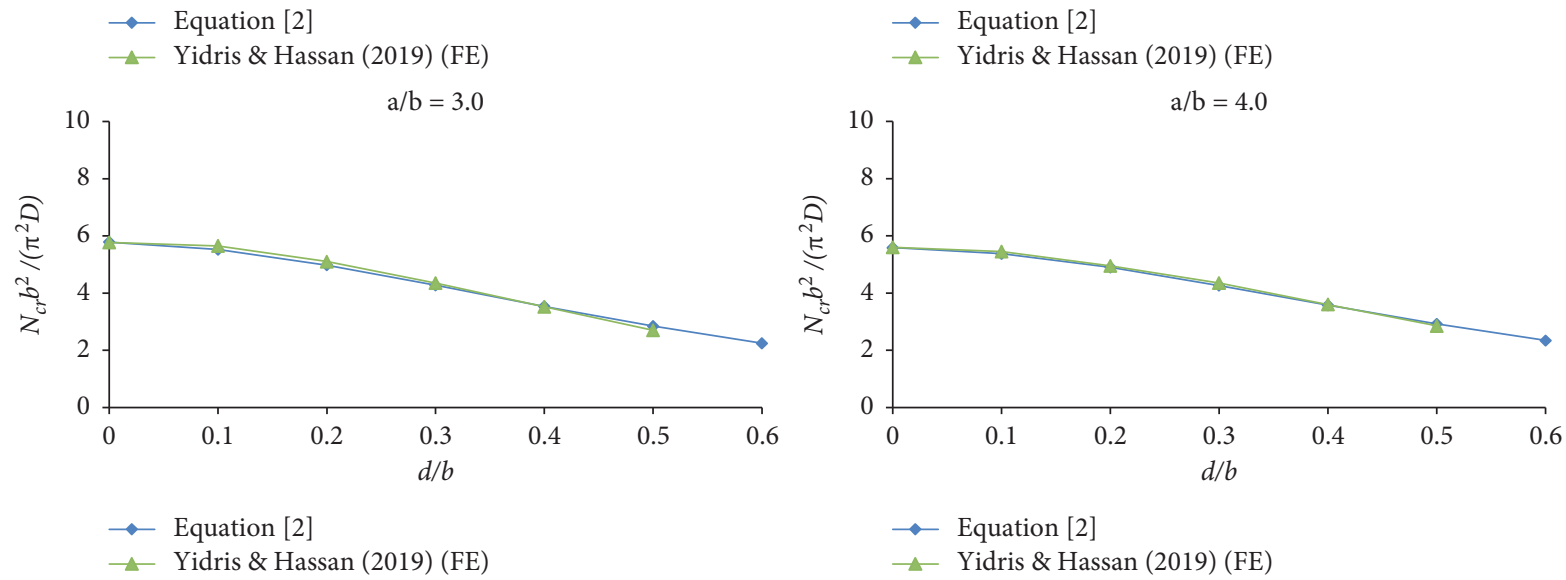

FIGURE 3: Normalized buckling loads for SSSS rectangular plates with central circular perforation exposed to shear loads. 

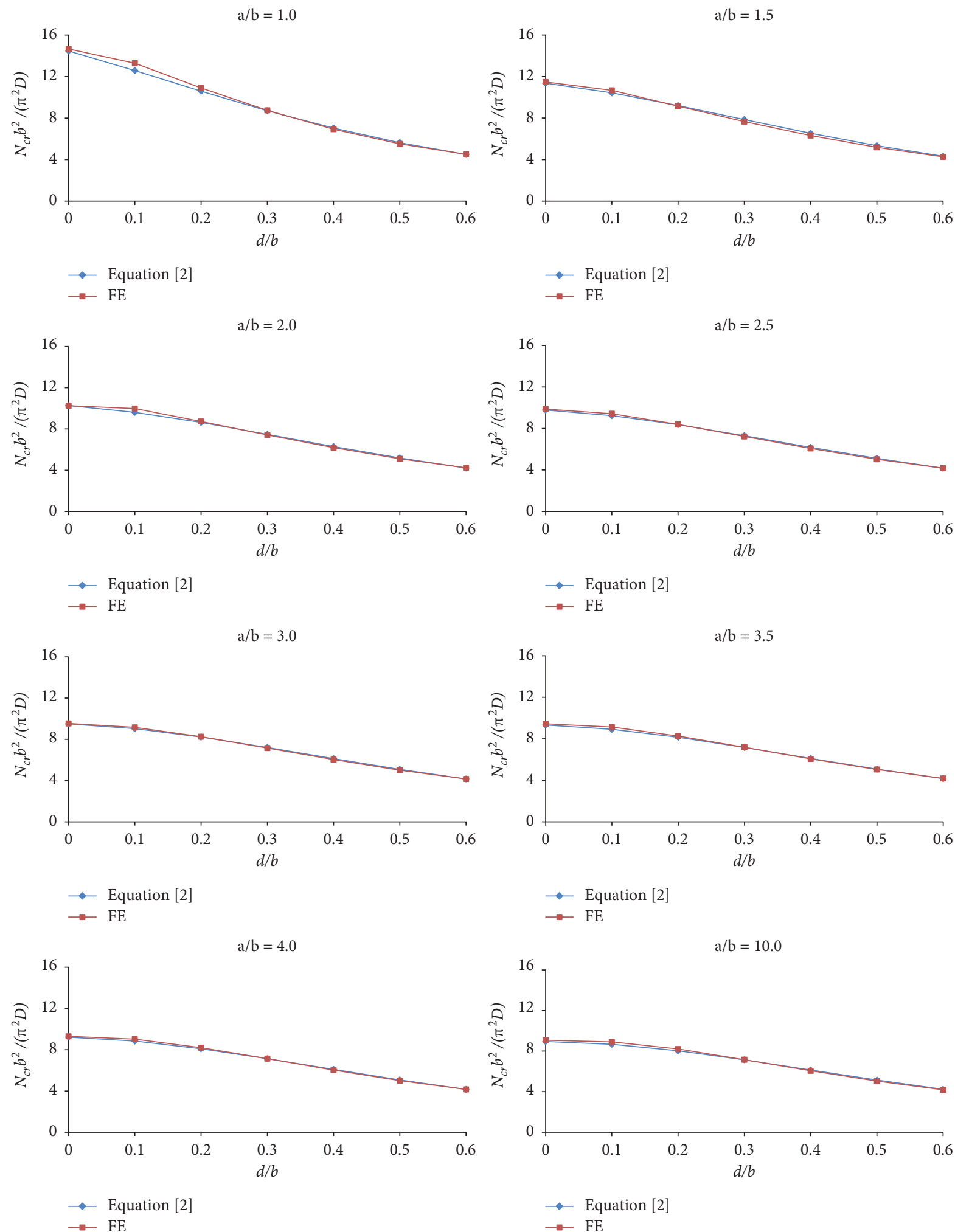

FIGURE 4: Normalized buckling loads for CCCC rectangular plates with central circular perforation exposed to shear loads.

percentage as shown in Figure 7. The normalized critical loads for each pattern and the percentage of cutout areas are shown in Figure 8. The figure shows that a refinement further than nine holes has almost no effect on the results.
Also, it can be seen that the shear buckling loads for plates containing a single central perforation are lower than that of plates with more perforations and of an equal total cutout area. 


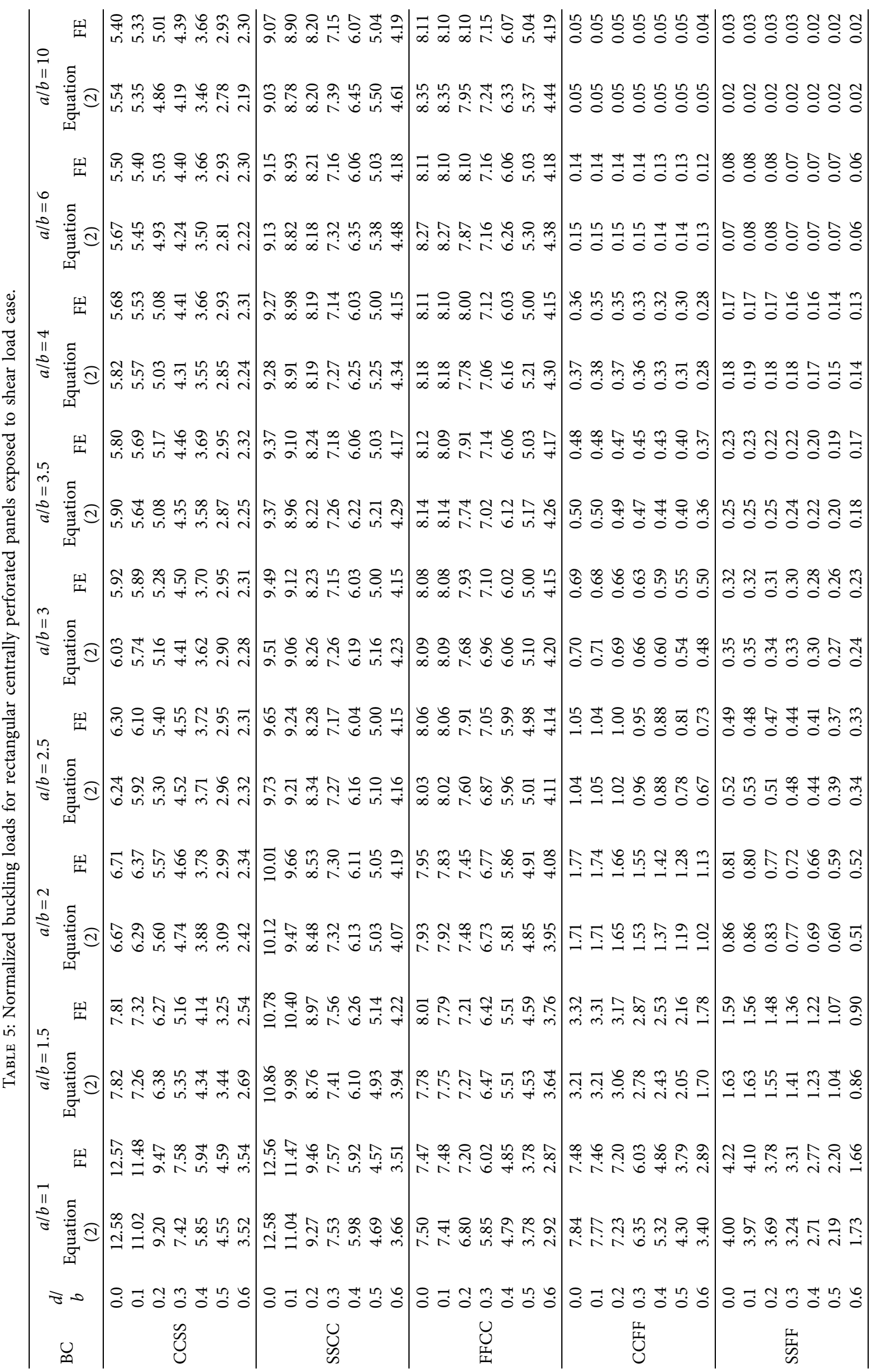




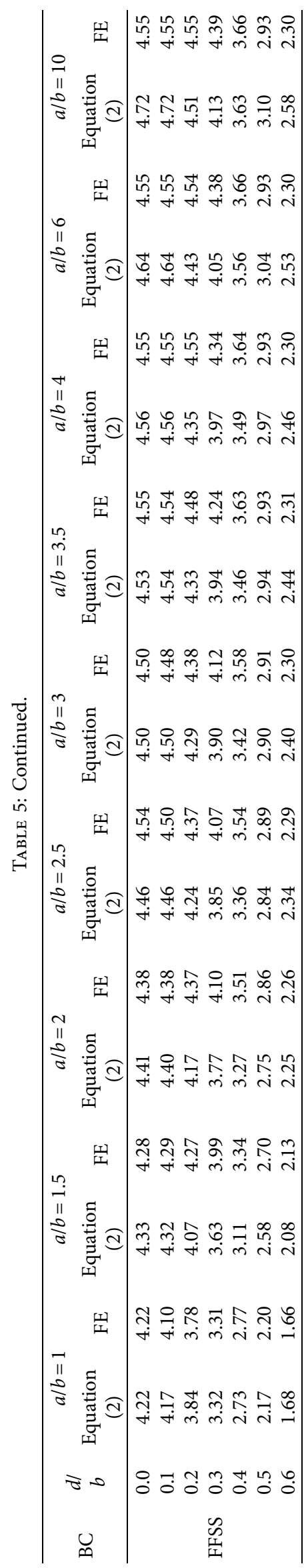



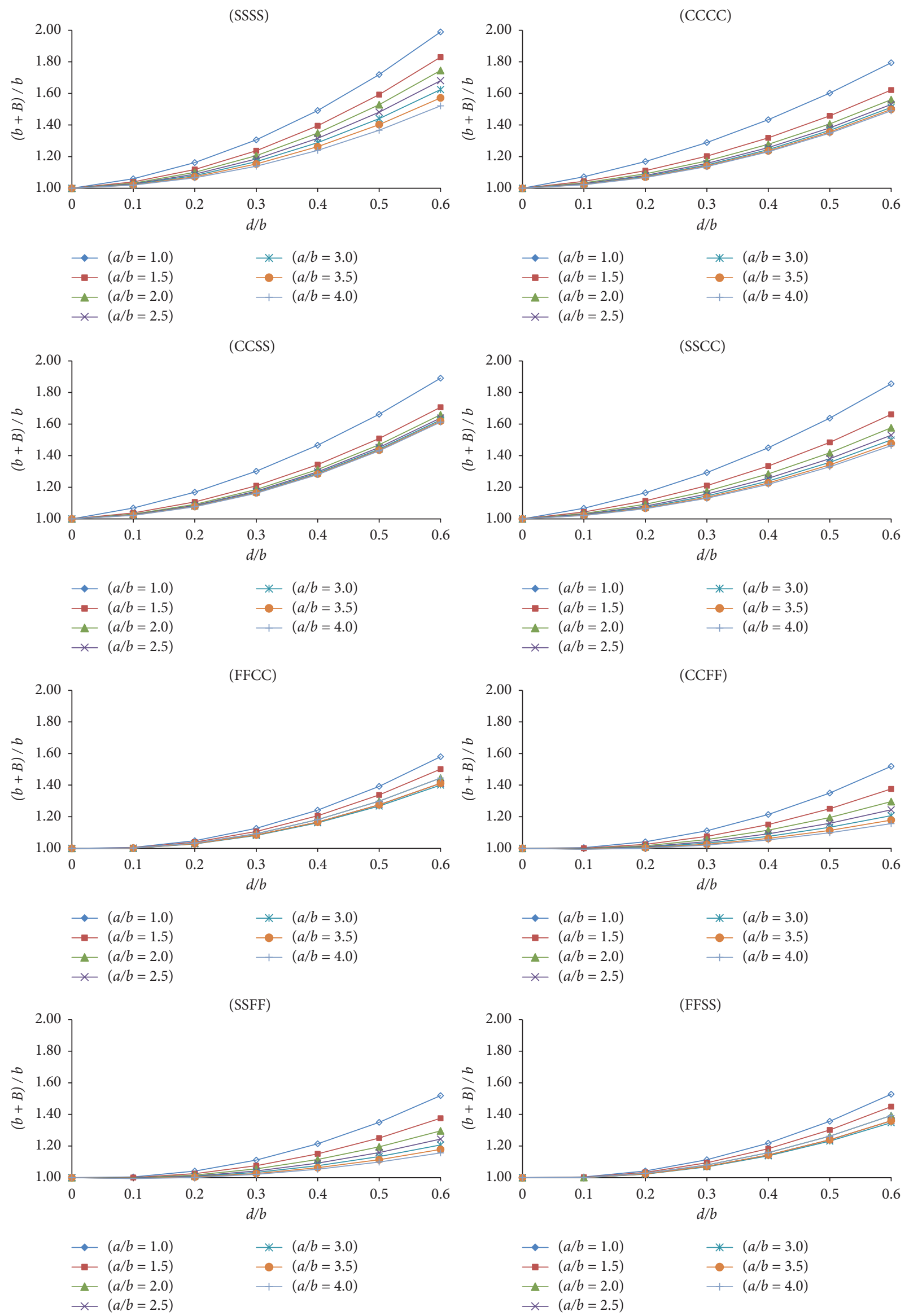

FIGURE 5: The proportion of the increase of the perforated rectangular plate width with different boundary conditions exposed to shear loads. 


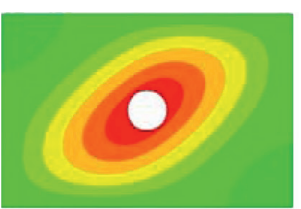

SSSS

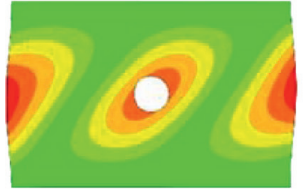

FFCC

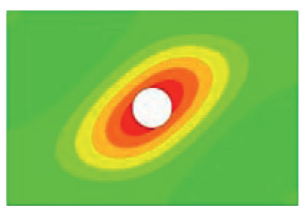

CCCC

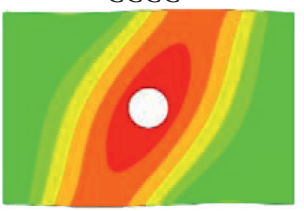

CCFF

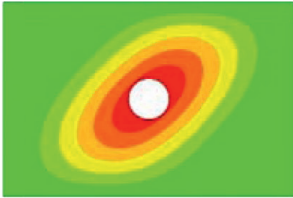

CCSS

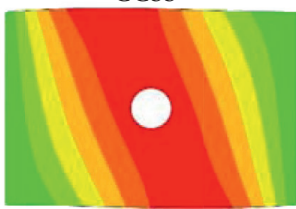

SSFF
U, Magnitude

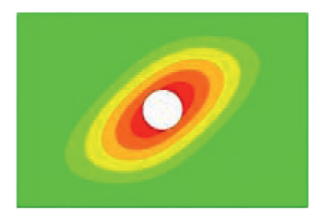

SSCC

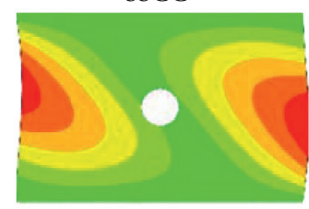

FFSS

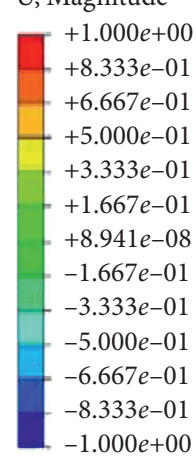

FiguRE 6: First mode shape for rectangular plate with different boundary conditions $(a / b=1.5, d / b=0.2)$ exposed to shear load.

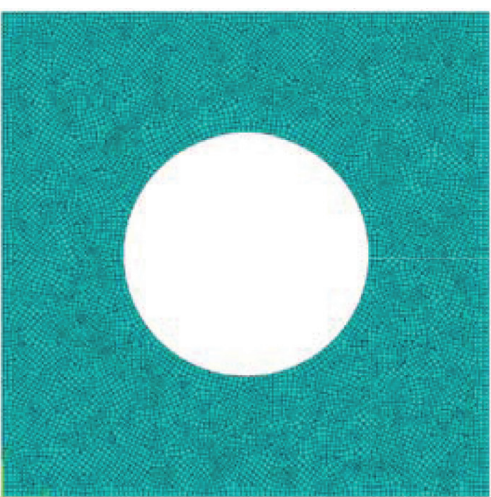

No of holes $=1$

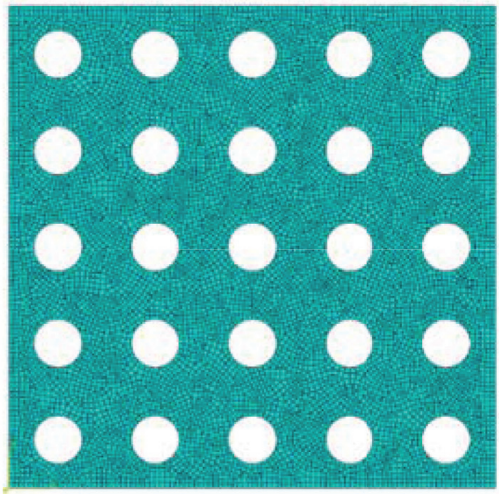

No of holes $=25$

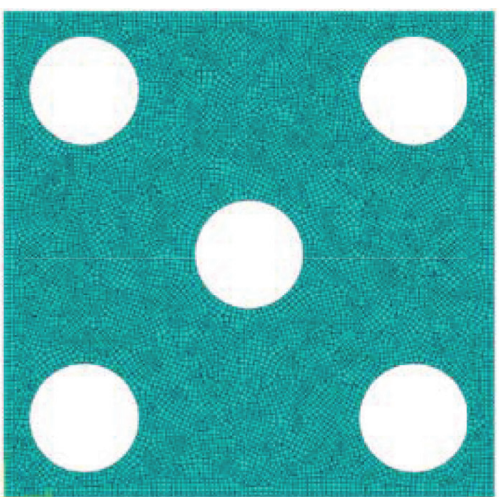

No of holes $=5$

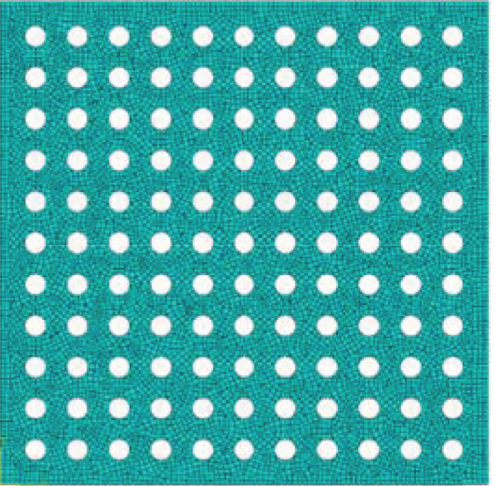

No of holes $=121$

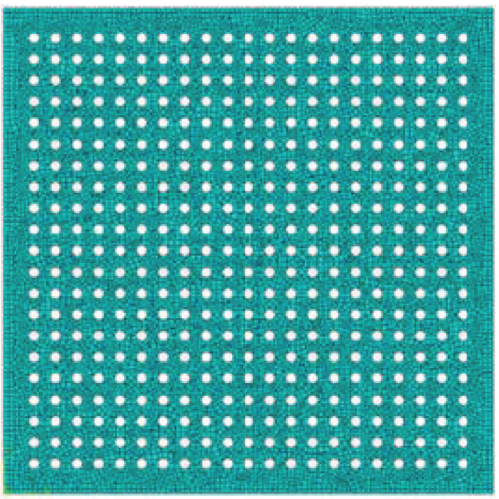

No of holes $=441$

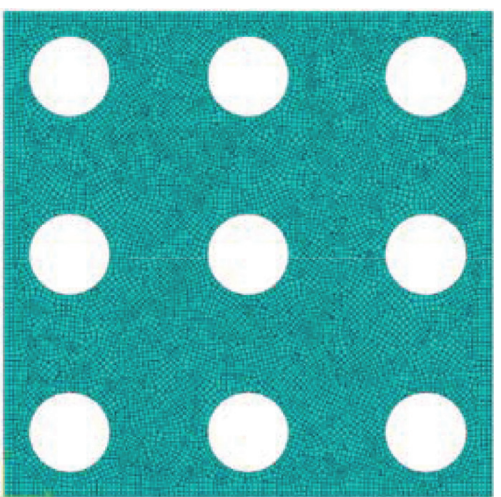

No of holes $=9$

000000000000000 0.000000000000 00000000000000 0.000 .0000000000 0.0000000000000 000000000000000 0,000000000000 000000000000000 000000000000000 0.0000 .000 .000 - 00.000000600 00000.000000000 .0000000000000 000000000000000 0.0000000000000

No of holes $=225$

Figure 7: Perforation patterns considered. 


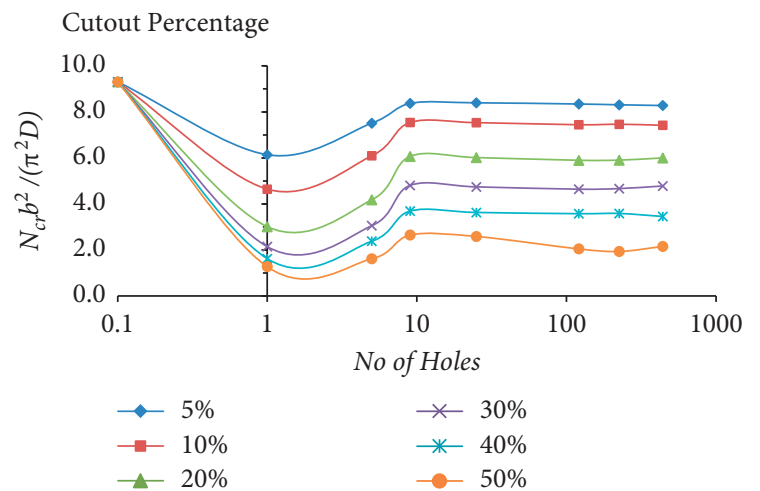

(a)

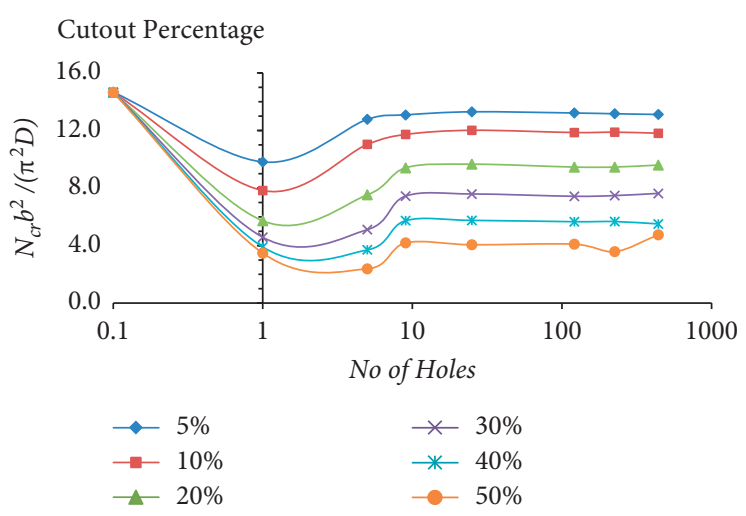

(b)

FIGURE 8: Normalized buckling loads for (a) SSSS and (b) CCCC square plates with various perforation patterns exposed to shear loads.

TABLE 6: Minimum critical temperature parameter $\propto T \times 10^{3}$ of square simply supported isotropic plate (SSSS) $\left(a / b=1, \propto=1 \times 10^{-6}\right.$, $E=200 \mathrm{GPa}$, and $\nu=0.3$ ).

\begin{tabular}{|c|c|c|c|c|c|c|c|c|}
\hline $\mathrm{BC}$ & $a / h$ & Reference [17] & Reference [20] & Reference [18] & Reference [19] & Reference [15] & Present FE & Present equation (2) \\
\hline \multirow{3}{*}{ SSSS } & 10 & 12.23 & 11.83 & & 11.83 & & 11.06 & 12.64 \\
\hline & 20 & 3.136 & 3.109 & & 3.089 & & 2.99 & 3.16 \\
\hline & 100 & 0.1265 & 0.1265 & 0.1265 & 0.1271 & & 0.1256 & 0.1264 \\
\hline CCCC & 100 & & & & & 0.34 & 0.335 & 0.337 \\
\hline
\end{tabular}

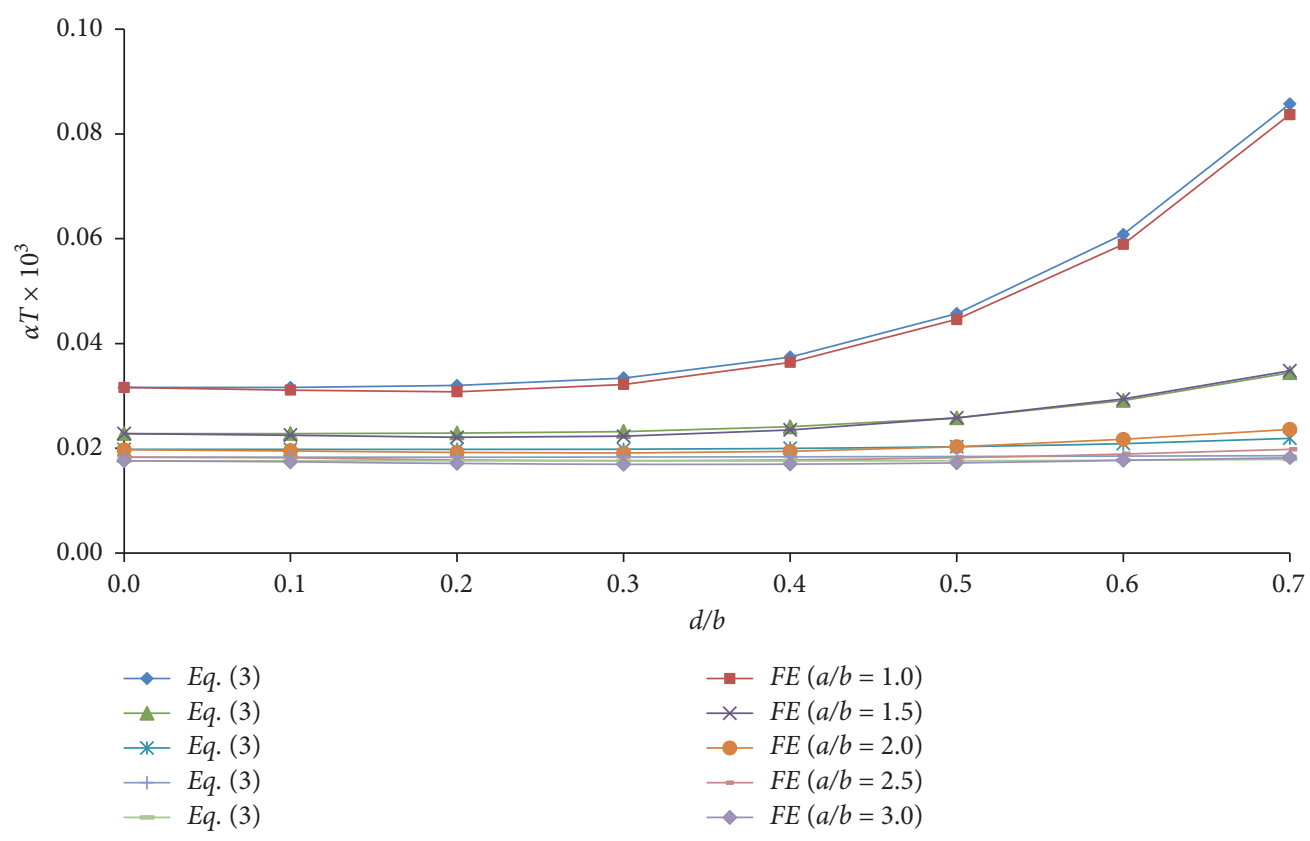

FIGURE 9: Minimum critical temperature parameter $\propto T \times 10^{3}$ of the simply central perforated isotropic plate (SSSS) $\left(\propto=1 \times 10^{-6}\right.$, $E=200 \mathrm{GPa}$, and $\nu=0.3$ ).

3.2. Thermal Buckling of Perforated Isotropic Plates. The minimum critical temperature of the perforated panels was predicted using the proposed semiempirical equations as listed in the preceding section (see equation (3) and Table 2). Thermal buckling analysis of the rectangular panels with circular/central cutouts was carried out using the nonlinear FE program (ABAQUS) as previously described. The results of the semiempirical equation (see equation (3)) were compared to findings from the literature and the nonlinear FE's results. The proposed equations for predicting minimum thermal buckling temperatures are shown to be effective in the various applications detailed below. In this study, the width dimensions $(b)$ of the panels were chosen to be $(b=2000 \mathrm{~mm})$. Herein, five different length-to-width ratios $(a / b)$ were used for the panels $(a / b=1.0, a / b=1.5, a /$ $b=2.0, a / b=2.5$, and $a / b=3.0)$. The panels' thickness was 


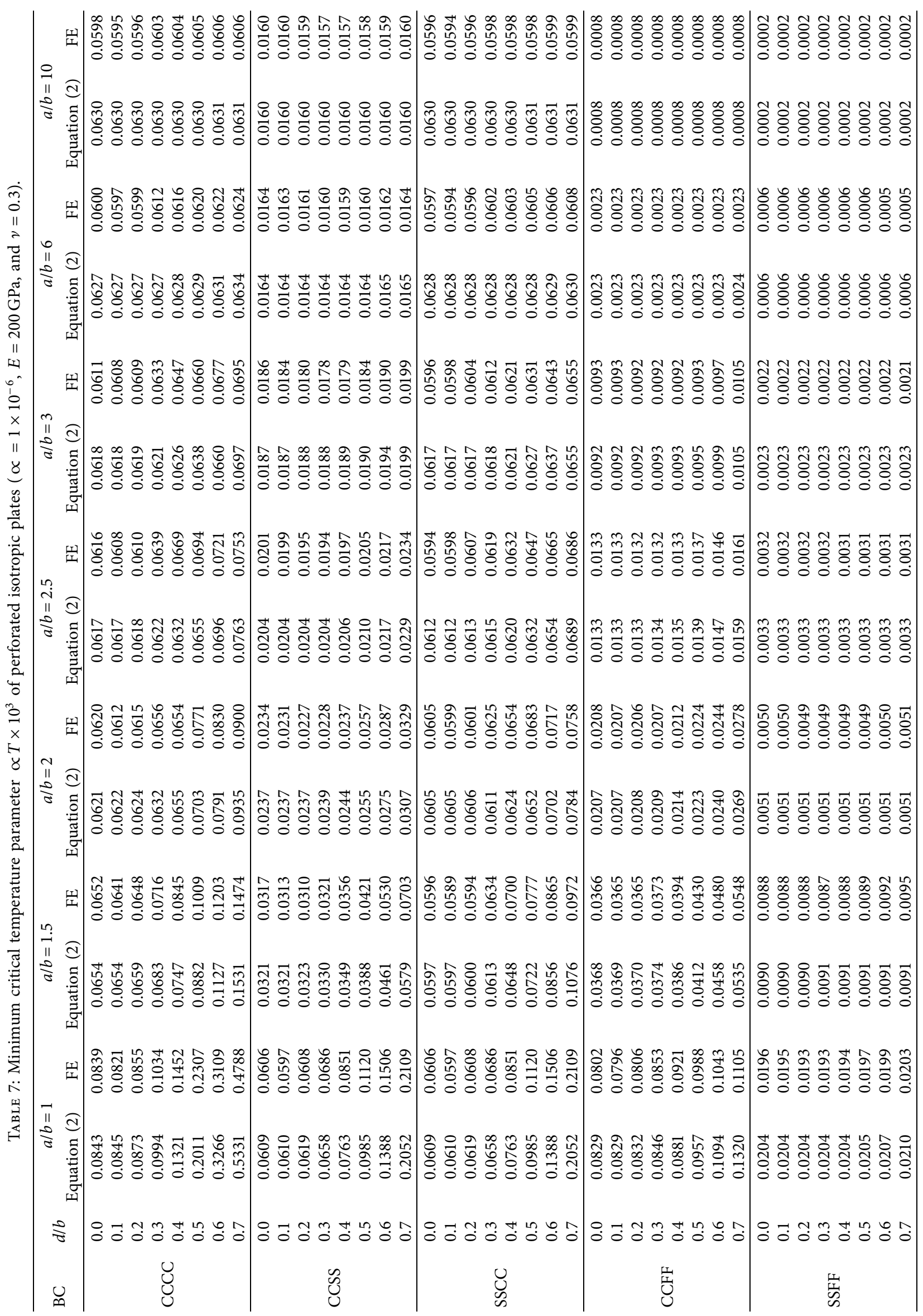




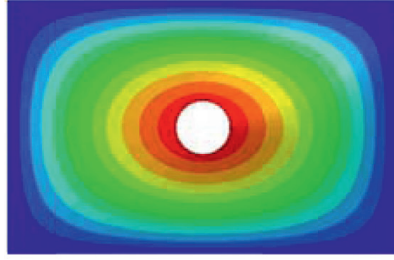

SSSS

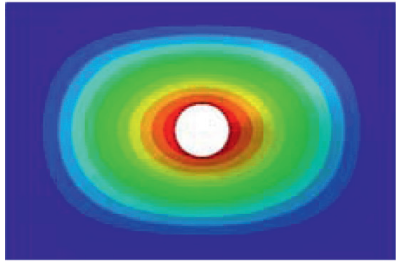

CCSS

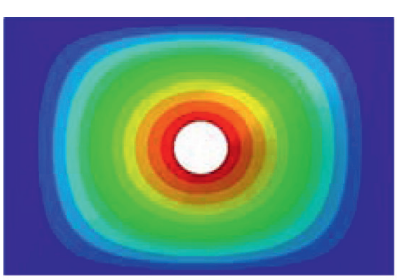

CCSS

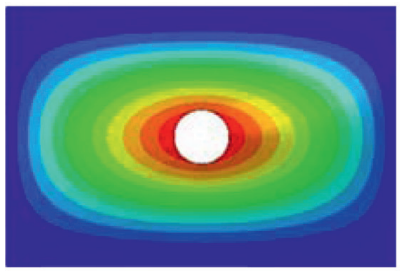

SSCC

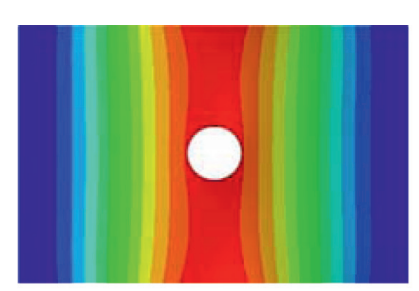

CCFF

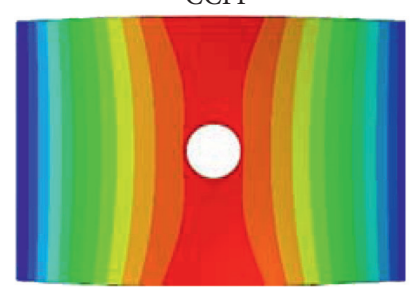

SSFF
U, Magnitude

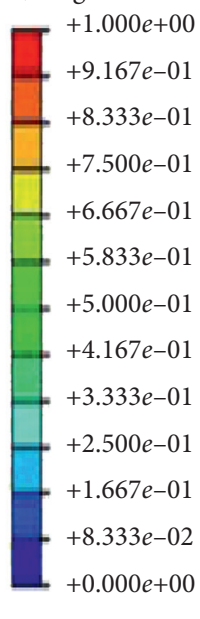

FIGURE 10: First mode shape for rectangular plate with different boundary conditions $(a / b=1.5, d / b=0.2)$ exposed to thermal load.

chosen to be $h=10 \mathrm{~mm}$. Different values for cutout diameters $(d)$ were used in this study. Thermal expansion coefficient $\alpha=1 \times 10^{-6} / \mathrm{K}$. Poisson's ratio $=0.3$ and Young's modulus $E=200 \mathrm{GPa}$ were chosen as isotropic material parameters (structural steel plate).

Table 6 shows the critical temperatures for square $(a /$ $b=1.0$ ) simply supported plate case (SSSS) and clamped supported case (CCCC) subjected to thermal loads. These tables summarize the available findings from the literature, as well as the suggested empirical equation (see equation (3)) and the FE outcomes. Figure 9 shows the critical temperatures for simply supported perforated rectangular isotropic plates (SSSS) with different lengthto-width $(a / b)$ proportions, while Table 7 shows the critical temperatures for perforated rectangular panels having CCCC, CCSS, SSCC, CCFF, and FFSS boundary conditions.

From Tables 6 and 7 and Figure 9, it is clear that the predicted results using equation (3) are very close to the results obtained from the FE and the literature. The results show that the thermal buckling loads increase as the cutout dimensions increase for all boundary conditions of the rectangular plates with different percentages. Furthermore, the effect of the perforation sizes is more crucial for small length to width proportions, as can be seen in Figure 9 and Table 7. It can be also noted from the results that the perforation dimensions, particularly for large cutout dimensions, have a significant impact on increasing the thermal buckling loads for square plates. It can be explained that with stronger boundary conditions and larger hole size, most of the thermal stresses are carried by the narrow strip of the plate along with the plate's supports, resulting in an increase in buckling resistance. The first mode shape for an isotropic rectangular panel with different boundary conditions exposed to thermal loads $(a / b=1.5, d / b=0.2)$ is shown in Figure 10. The boundary conditions have a strong influence on the variances in the displacement contour lines (mode shape).

\section{Conclusions}

The global buckling loads of isotropic perforated rectangular panels with different boundary conditions exposed to shear and thermal loading are calculated using semiempirical closed-form formulas in this study. Comparative results with semiempirical formulations were obtained using a nonlinear FE model. The findings of the suggested semiempirical models, the accessible outcomes of the literature, and the outcomes from the FE model were all in good agreement, confirming the validity and usability of the semiempirical formulas. The proposed equations will make it possible to compute the buckling loads for isotropic perforated rectangular panels rapidly and accurately. In the perspective of the buckling analysis of circular perforated rectangular plates, this study provides a wealth of information that may be used in comparisons and future research. The following are some conclusions that can be drawn based on the numerical outcomes:

(i) The impact of the perforation on the shear buckling load is increased when the perforation diameters are increased for all rectangular plates exposed to shear loads

(ii) The perforation's influence on the buckling load is reduced when the plate length/width proportion (a) $b$ ) is increased

(iii) The boundary conditions significantly impact the proportion of the reduction in shear buckling loads caused by the cutout

(iv) The highest shear and thermal buckling resistance was achieved for plates having CCCC boundary conditions, while the lowest shear buckling resistance was achieved for plates having SSFF boundary conditions

(v) Increasing the size of the perforation reduces the shear buckling load and increases the thermal buckling load for all the boundary conditions mentioned in this study 
(vi) The shear buckling load of a plate with a single central perforation is lower than that of a plate with more perforations and of an equal total cutout area

\section{Data Availability}

The data that support the findings of this study are available from the author upon reasonable request.

\section{Conflicts of Interest}

The author declares no conflicts of interest.

\section{References}

[1] K. M. El-sawy and A. S. Nazmy, "Effect of aspect ratio on the elastic buckling of uniaxially loaded plates with eccentric holes," Thin-Walled Structures, vol. 39, no. 12, pp. 983-998, 2001.

[2] H. Al Qablan, H. Katkhuda, and H. Dwairi, “Assessment of the buckling behavior of square composite plates with circular cutout subjected to in-plane shear," Jordan Journal of Civil Engineering, vol. 3, pp. 184-195, 2009.

[3] C. H. Pham, "Shear buckling of plates and thin-walled channel sections with holes," Journal of Constructional Steel Research, vol. 128, pp. 800-811, 2017.

[4] S. Abolghasemi, H. Eipakchi, and M. Shariati, "An analytical solution for buckling of plates with circular cutout subjected to non-uniform in-plane loading," Archive of Applied Mechanics, vol. 89, no. 12, pp. 2519-2543, 2019.

[5] H. A. Qablan, S. Rabab'ah, B. A. Alfoul, and O. A. Hattamleh, "Semi-empirical buckling analysis of perforated composite panel," Mechanics Based Design of Structures and Machines, pp. 1-18, 2020.

[6] M. Aydin Komur and M. Sonmez, "Elastic buckling of rectangular plates under linearly varying in-plane normal load with a circular cutout," Mechanics Research Communications, vol. 35, no. 6, pp. 361-371, 2008.

[7] D. Kumar and S. B. Singh, "Effects of boundary conditions on buckling and postbuckling responses of composite laminate with various shaped cutouts," Composite Structures, vol. 92, no. 3, pp. 769-779, 2010.

[8] N. Yidris and M. N. Hassan, The Effects of Cut-Out on ThinWalled Plates, Woodhead Publishing, Sawston, Cambridge, UK, 2019.

[9] B. Mohammadzadeh, E. Choi, and W. J. Kim, "Comprehensive investigation of buckling behavior of plates considering effects of holes Comprehensive investigation of buckling behavior of plates considering effects of holes," Structural Engineering and Mechanics, vol. 68, pp. 261-275, 2018.

[10] H. Al-Qablan, H. Dwairi, N. Shatarat, T. Rosan, and T. AlQablan, "Stability analysis of composite panels with stiffeners and circular cutouts," Jordan Journal of Civil Engineering, vol. 4, pp. 119-131, 2010.

[11] G. Soni, R. Singh, and M. Mitra, "Buckling behavior of composite laminates (with and without cutouts) subjected to nonuniform in-plane loads," International Journal of Structural Stability and Dynamics, vol. 13, no. 8, pp. 1-20, 2013.

[12] J.-H. Kim, D.-H. Park, S.-K. Kim, J.-D. Kim, and J.-M. Lee, "Lateral deflection behavior of perforated steel plates: experimental and numerical approaches," Journal of Marine Science and Engineering, vol. 9, no. 5, p. 498, 2021.

[13] M. Aydin Komur, F. Sen, A. Ataş, and N. Arslan, "Buckling analysis of laminated composite plates with an elliptical/ circular cutout using FEM," Advances in Engineering Software, vol. 41, no. 2, pp. 161-164, 2010.

[14] S. A. M. Ghannadpour, A. Najafi, and B. Mohammadi, "On the buckling behavior of cross-ply laminated composite plates due to circular/elliptical cutouts," Composite Structures, vol. 75, no. 1-4, pp. 3-6, 2006.

[15] M. Amabili and M. R. S. Tajahmadi, "Thermal post-buckling of laminated and isotropic rectangular plates with fixed edges: comparison of experimental and numerical results," Proceedings of the Institution of Mechanical Engineers - Part C: Journal of Mechanical Engineering Science, vol. 226, no. 10, pp. 2393-2401, 2012.

[16] R. Kumar, L. S. Ramachandra, and B. Banerjee, "Semi-analytical approach for thermal buckling and postbuckling response of rectangular composite plates subjected to localized thermal heating," Acta Mechanica, vol. 228, no. 5, pp. 1767-1791, 2017.

[17] A. K. Noor and W. S. Burton, "Three-dimensional solutions for thermal buckling of multilayered anisotropic plates," Journal of Engineering Mechanics, vol. 118, no. 4, pp. 683-701, 1992.

[18] H. Ounis, A. Tati, and A. Benchabane, "Thermal buckling behavior of laminated composite plates: a finite-element study," Frontiers of Mechanical Engineering, vol. 9, no. 1, pp. 41-49, 2014.

[19] X. Zhao, Y. Y. Lee, and K. M. Liew, "Mechanical and thermal buckling analysis of functionally graded plates," Composite Structures, vol. 90, no. 2, pp. 161-171, 2009.

[20] H. Matsunaga, "Thermal buckling of cross-ply laminated composite and sandwich plates according to a global higherorder deformation theory," Composite Structures, vol. 68, no. 4, pp. 439-454, 2005.

[21] S. Timoshenko and J. Gere, Theory of Elastic Stability, Courier Corporation, New York, NY, USA, 2nd edition, 1961.

[22] R. Abbott, Analysis and Design of Composite and Metallic Flight Vehicle Structures, Abbott Aerospace SEZC Ltd., Collingwood, ON, Canada, 1st edition, 2016.

[23] K. William, "Thermal buckling analysis of rectangular panels subjected to humped temperature profile heating," Structural Mechanics, vol. 57, pp. 1-34, 2004. 